\title{
Thermoluminescence response of sodalime glass irradiated with photon and electron beams in the 1-20 Gy range
}

\author{
A. Bartolotta ${ }^{\mathrm{a}, *}$, M. Brai ${ }^{\mathrm{b}, \mathrm{c}}$, V. Caputo ${ }^{\mathrm{d}}$, M.C. D’Oca ${ }^{\mathrm{a}}$, A. Longo ${ }^{\mathrm{b}, \mathrm{c}}$, M. Marrale $^{\mathrm{b}, \mathrm{c}}$ \\ ${ }^{a}$ Dipartimento Farmacochimico, Tossicologico e Biologico, via Archirafi 32, 90123 Palermo, Italy \\ ${ }^{\mathrm{b}}$ Dipartimento di Fisica e Tecnologie Relative, Università di Palermo, Viale delle Scienze, Ed. 18, 90128 Palermo, Italy \\ ${ }^{\mathrm{c}}$ Gruppo V, INFN, Sezione di Catania, Catania, Italy \\ ${ }^{\mathrm{d}}$ Unità Operativa Complessa Fisica Sanitaria, ARNAS, Palermo, Italy
}

\section{A R T I C L E I N F O}

\section{Article history:}

Received 22 November 2010

Received in revised form

25 January 2011

Accepted 5 March 2011

\begin{abstract}
A B S T R A C T
The thermoluminescence response of a watch commercial glass was studied after irradiation with photons and electrons, in the range 1-20 Gy, of interest in accidental dosimetry; a linear response was obtained with both beams. This result, together with the satisfactory time stability of the thermoluminescence signal, indicates this glass as a potential material for retrospective dosimetry applications.
\end{abstract}

(c) 2011 Elsevier Ltd. All rights reserved.

\section{Keywords:}

Thermoluminescence

Soda lime watch glasses

Retrospective dosimetry

\section{Introduction}

Various authors have recently proposed the use of glass samples as ionizing radiation detectors in different fields (Correcher et al., 2009; Engin et al., 2010; Balogun et al., 2003); above all, there is an increasing interest on glasses which can be in contact or very close to exposed persons and can be used as emergency dosimeters. Glass has valuable properties such as easy handling, chemical inertness and rigidity. The radiation induced effects in the irradiated glass can be indeed employed for dose evaluation, by means of two physical techniques, i.e. thermoluminescence (TL) (Teixeira et al., 2008; Narayan et al., 2008) and electron spin resonance (ESR) (Gancheva et al., 2006; Teixeira et al., 2005).

In the present work, the TL response of a commercial watch glass was investigated using glass chips obtained by a simple cutting procedure. In particular, samples of disk-shaped sodalime watch commercial glass have been irradiated with a 6 MV photon beam and a $10 \mathrm{MeV}$ electron beam, both used in radiation therapy. An analysis of the glow curve has been carried out in order to characterize the TL signal related to irradiation. For each beam, a linear dose response behavior has been found and the dependence on beam quality is under investigation. Furthermore, a high

\footnotetext{
* Corresponding author. Tel.: +91 6167210; fax: +916230923.

E-mail address: antonio.bartolotta@unipa.it (A. Bartolotta).
}

homogeneity has also been observed and the signal fading has been analyzed. These results suggest the use of watch glass for accidental dosimetry; work is in progress to investigate the possibility of applying the single aliquot regenerative dose (SAR) protocol (Hong et al., 2006) or the multiple aliquot additive dose (MAAD) method (Bassinet et al., 2006) for dose reconstruction.

\section{Materials and methods}

\subsection{Sample preparation}

Experiments were carried out with a commercial watch glass, provided by Ghignone U. \& Co. (Milan, Italy), that is composed of the following main elements (weight percentage): $\mathrm{SiO}_{2}(72.5 \%)$, $\mathrm{Na}_{2} \mathrm{O}(12.7 \%), \mathrm{CaO}(5.0 \%), \mathrm{MgO}(6.0 \%), \mathrm{Al}_{2} \mathrm{O}_{3}(1.6 \%)$ and is classified as sodalime glass (Longo et al., 2010). Samples of disk-shaped glasses (diameter $30 \mathrm{~mm}$, thickness $2.0 \mathrm{~mm}$ ) were irradiated as a whole; after irradiation they were cut in little pieces (chip characteristics: size usually $3 \times 3 \mathrm{~mm}^{2}$, weight in the range $30-100 \mathrm{mg}$ ) suitable to be placed on the planchet of the Harshaw 3500 TL reader. No sample treatment was performed before TL measurements.

To study the time stability of the TL signal, chips cut from irradiated disks were read after increasing time intervals of storage under the following conditions: i) dark; ii) a mixture of natural and artificial light (laboratory conditions). The average temperature was in each case $(20 \pm 5)^{\circ} \mathrm{C}$. 


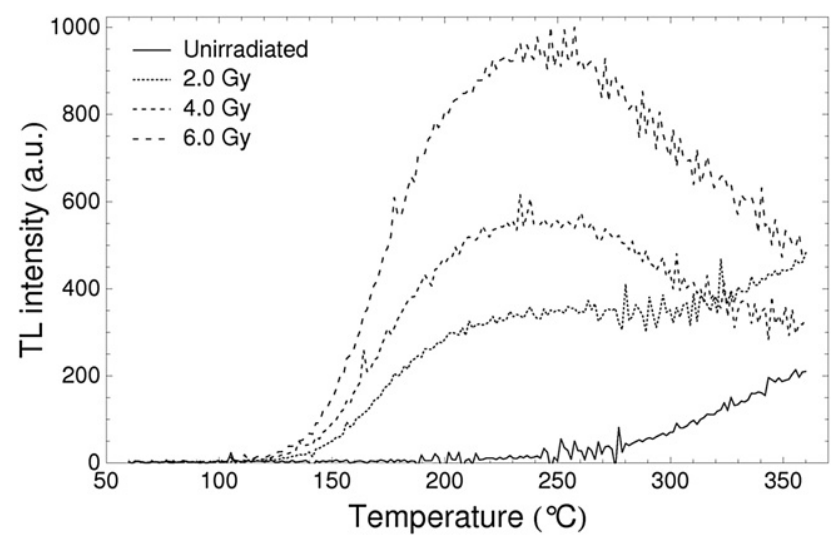

Fig. 1. Glow curves of glass chips unirradiated or irradiated with the electron beam at 2-4-6 Gy.

\subsection{Irradiation}

Irradiations were carried out with a $6 \mathrm{MV}$ photon beam and with a $10 \mathrm{MeV}$ electron beam, both produced by the Linear Accelerator Siemens Primus Low used in the Radiotherapy Unit of the ARNAS, Palermo. The samples were irradiated in the dose range between 1 and $20 \mathrm{~Gy}$ inside a perspex phantom. Dose values were calculated using the dose rate previously measured with a reference ionization chamber and the irradiation time, with an overall uncertainty of about $3 \%$.

\subsection{Thermoluminescence measurements}

All the glow curves were recorded with an Harshaw $3500 \mathrm{TL}$ reader; the instrumental parameters were set as follows: voltage $500 \mathrm{~V}$, preheat at $60^{\circ} \mathrm{C}$ for $10 \mathrm{~s}$, heating rate $5^{\circ} \mathrm{C} / \mathrm{s}$, final temperature $360^{\circ} \mathrm{C}$. A filter to partially reject black body radiation was used.

\section{Results and discussion}

\subsection{Glow curve features}

The glow curve of the irradiated glass shows a single peak, centered at about $230^{\circ} \mathrm{C}$, regardless the beam and the dose. As an example, Fig. 1 shows the glow curves of single chips cut from glass disks, previously unirradiated or irradiated with the electron beam at 2-4-6 Gy. The glow curve of the unirradiated sample overlaps with the planchet emission curve, indicating the absence of

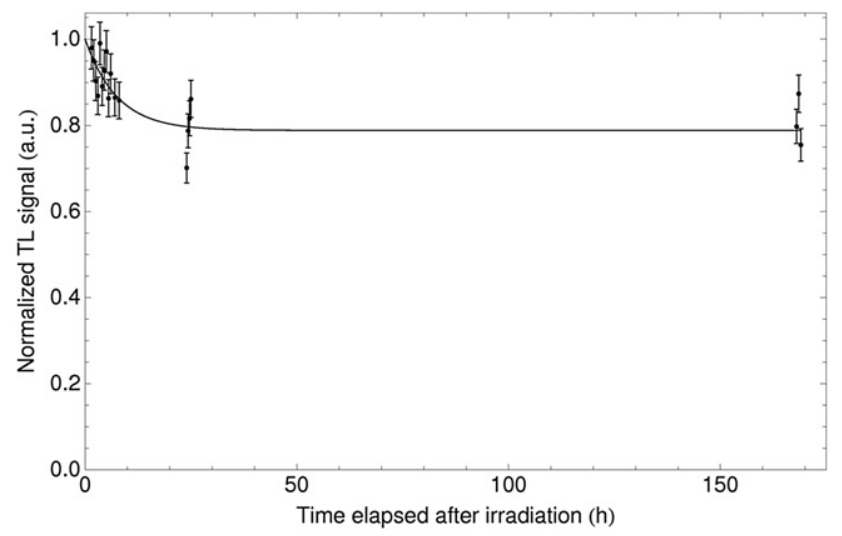

Fig. 2. TL signal fading of glass irradiated at $2 \mathrm{~Gy}$ (laboratory conditions).
Table 1

Homogeneity test on chips cut from different glass disks irradiated at $15 \mathrm{~Gy}$.

\begin{tabular}{llcl}
\hline chip & mass $/ \mathrm{mg}$ & TL signal $/ 10^{4}$ & TL/mass \\
\hline A & 31.8 & 9.84 & 3094 \\
B & 60.4 & 17.7 & 2931 \\
C & 41.0 & 12.5 & 3049 \\
D & 45.9 & 14.8 & 3224 \\
E & 39.2 & 11.9 & 3036 \\
\hline
\end{tabular}

a significant contribution due to natural radiation. For each recorded glow curve, the integrated TL signal was calculated as the sum of the counts recorded by the TL apparatus in the temperature range $230^{\circ} \mathrm{C} \pm 90^{\circ} \mathrm{C}$, and normalized to the chip mass. The background signal due to the planchet was always subtracted.

\subsection{Time stability of the TL signal}

The time trend of the mass normalized TL signal was analyzed: two disks were irradiated at 2 and 5 Gy with photons, and subsequently cut in twenty-five chips each, stored as described in Section 2.1. For both storage conditions, the main reduction of the signal was observed to occur in the first $50 \mathrm{~h}$, when it turned out to be between $70 \%$ and $80 \%$ of the original signal; afterward, the signal remained almost constant even after about one week. As an example, Fig. 2 shows the TL signal as a function of storage time (laboratory conditions) after irradiation at $2 \mathrm{~Gy}$. The knowledge of the fading trend could be useful for application in retrospective dosimetry.

\subsection{Homogeneity analysis}

A check on the homogeneity of the TL response of chips taken from different glass disk samples, irradiated at the same dose, was first carried out. The glass chips differed among each other in size and mass; the dependence of the TL signal on the chip mass was therefore studied. Table 1 shows the results obtained with glass chips cut from disk samples irradiated at 15 Gy with the 6 MV photon beam; despite a variation in mass up to a factor of two, the TL signal normalized to the mass shows a coefficient of variation of only $3.5 \%$; similar results were obtained with all samples, regardless the dose.

\subsection{TL signal vs. dose}

To study the dose dependence of the TL signal, glass disk samples were irradiated, each one at a specific dose up to $20 \mathrm{~Gy}$,

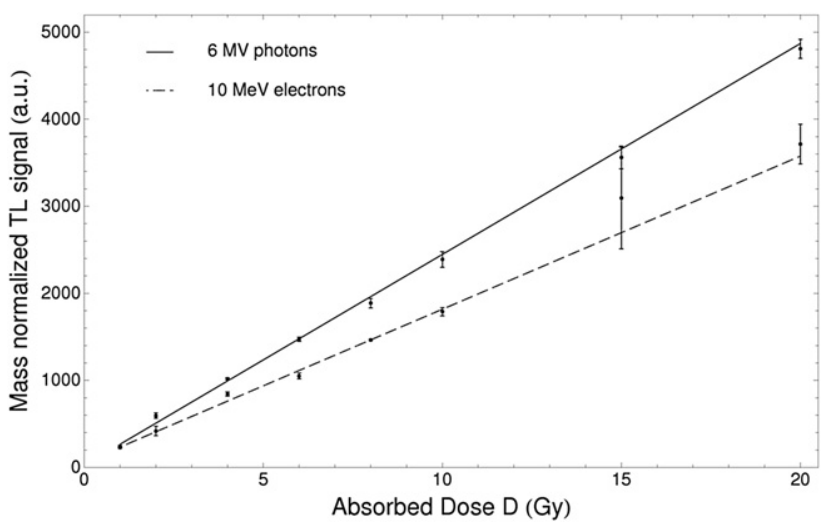

Fig. 3. TL vs dose response (experimental data and best fitting straight line). 
Table 2

Best fitting parameters for the TL signal vs dose $\mathrm{D}$ analysis $(\mathrm{TL}=\mathrm{a}+\mathrm{bD})$.

\begin{tabular}{llll}
\hline & $\mathrm{a}$ & $\mathrm{b}$ & $\mathrm{R}^{2}$ \\
\hline $6 \mathrm{MV}$ photons & $23 \pm 11$ & $242 \pm 4$ & 0.998 \\
$10 \mathrm{MeV}$ electrons & $57 \pm 16$ & $176 \pm 3$ & 0.999 \\
\hline
\end{tabular}

with the beams used in this work; three chips were taken from each disk, and the corresponding glow curves were recorded. For each chip, the TL signal was obtained as described in Section 3.1.

For each dose D, the average TL signal of the three chips was used to study the dose response behavior. The Lowest Detectable Signal (LDS) was evaluated as the average TL signal of ten unirradiated chips plus three standard deviation; the TL signal of the chips irradiated at the lowest dose (1 Gy) was more than four times the LDS.

Fig. 3 reports the best fitting calibration straight lines ( $\mathrm{TL}=$ $\mathrm{a}+\mathrm{bD}$ ) obtained for watch glasses exposed to $6 \mathrm{MV}$ photons and $10 \mathrm{MeV}$ electrons, respectively. A good linear response was observed for each beam, as also confirmed by the $\mathrm{R}^{2}$ correlation coefficient. Table 2 shows the fitting parameters. The higher sensitivity observed for photons with respect to electrons could be due to the different Linear Energy Transfer (LET) of the two beams.

\section{Conclusions}

The results discussed in this paper show that the watch glass investigated in this work is potentially a good candidate for retrospective dosimetry in radiological emergency, due to its crucial features:

- The watch glasses are in contact with the exposed person

- Good physical and chemical features: chemical inertness, insolubility, rigidity, small size

- Easy sample preparation

- Homogeneity of the TL response

- Linearity of the TL response

- Time stability
Further analyses are required with other radiation beams, to better study the dependence of the TL response on the LET. The applicability of a method (SAR or MAAD) for retrospective dose evaluation will be investigated, even after irradiation of the glass in a mixed radiation field. To this aim, further study on the homogeneity, fading under various storage conditions and sensitivity stability are in progress.

\section{Acknowledgments}

This research was partially supported by Istituto Nazionale di Fisica Nucleare (INFN), DOSSIER Project, and by Università di Palermo (Fondi di Ricerca Scientifica di Ateneo).

\section{References}

Balogun, F.A., Ogundare, F.O., Fasasi, M.K., 2003. TL response of sodalime glass at high doses. Nucl. Instr. Met. A 505, 407-410.

Bassinet, C., Mercier, N., Miallier, D., Pilleyre, T., Sanzelle, S., Valladas, H., 2006. Thermoluminescence of heated quartz grains: intercomparisons between SAR and multiple-aliquot additive dose techniques. Rad. Meas. 41, 803-808.

Correcher, V., Garcia-Guinea, Rivera, T., 2009. Thermoluminescence sensitivity of daily use materials. Radiat. Eff. Defects Sol 164, 59-67.

Engin, B., Ayda, C., Demirta, H., 2010. Study of the thermoluminescence dosimetric properties of window glass. Radiat. Eff. Defects Sol 165, 54-64.

Gancheva, V., Yordanov, N.D., Karakirova, Y., 2006. EPR investigation of the gamma radiation response of different types of glasses. Spectrochim. Acta A 63, $875-878$.

Hong, D.G., Kim, M.J., Choi, J.H., El-Faramawy, N.A., Göksu, H.Y., 2006. Equivalent dose determination of single aliquot regenerative-dose (SAR) protocol using thermoluminescence on heated quartz. Nucl. Instr. Meth. Phys. Res. B. 243, $174-178$.

Longo, A., Basile, S., Brai, M., Marrale, M., Tranchina, L., 2010. ESR response of watch glasses to proton beams. Nucl. Instr. Meth. Phys. Res. B. 268, 2712-2718.

Narayan, P., Vaijapurkar, S.G., Senwar, K.R., Kumar, D., Bhatnagar, P.K., 2008. Accidental gamma dose measurement using commercial glasses. Radiat. Prot. Dos $130,319-324$

Teixeira, M.I., Ferraz, G.M., Caldas, L.V.E., 2005. EPR dosimetry using commercial glasses for high gamma doses. Appl. Radiat. Isot 62, 365-370.

Teixeira, M.I., Da Costa, Z.M., Da Costa, C.R., Pontuschka, W.M., Caldas, L.V.E., 2008. Study of the gamma radiation response of watch glasses. Radiat. Meas. $43,480-482$ 